\title{
Caracterización en pre y poscosecha de dos cultivares de melón reticulado del tipo Oriental (Cucumis melo Grupo Cantalupensis)
}

\author{
Dominique Laínez y Christian Krarup ${ }^{1}$ \\ Departamento de Ciencias Vegetales, Facultad de Agronomía e Ingeniería Forestal \\ Pontificia Universidad Católica de Chile \\ Casilla 306-22, Santiago, Chile
}

\begin{abstract}
D. Laínez, and C. Krarup. 2008. Pre- and post-harvest characterization of two reticulate melon cultivars of the Oriental type (Cucumis melo Group Catalupensis). Cien. Inv. Agr. 35(1): 59-66. To characterize the pre- and post-harvest performance of Oriental-type cantaloupes (Cucumis melo Group Cantalupensis), cultivars Emerald and Glamour were grown following the common culture practices of Chile. Both cultivars exhibited vigorous plant growth and high productivity, but they produce fruits with different characteristics. Fruits of melon cv. Emerald slip when ripe, were medium sized $(1.0 \mathrm{~kg})$ and oval-shaped, with a green epidermis, fine and sparse superficial netting, and green to whitish pulp with $10.3 \%$ mean content of total soluble solids (TSS). In contrast, fruits of cv. Glamour did not slip when ripe, were almost round-shaped, medium sized $(1.1 \mathrm{~kg})$, and had a greyish yellow epidermis, coarse and dense netting, and a bright orange pulp with $11.4 \%$ mean TSS content. Yields were higher than for other cantaloupe cultivars, reaching $42.4 \mathrm{t}^{-h a^{-1}}$ in cv. Glamour and $53.2 \mathrm{t} \cdot \mathrm{ha}^{-1} \mathrm{in} \mathrm{cv}$. Emerald. Post-harvest behaviour was evaluated after 18 days of storage at 0 and $10^{\circ} \mathrm{C}$, and after three additional days at $20^{\circ} \mathrm{C}$. Results indicated a low sensitivity to chilling injury, particularly for Glamour fruits, which kept better at 0 than at $10^{\circ} \mathrm{C}$ with lower weight loss and a slower postharvest disease development, leading to a higher final quality. Overall, cv. Glamour is a cultivar with high potential for the fresh market and industry, showing more attractive characteristics, better storage, and higher quality than cv. Emerald.
\end{abstract}

Keywords: Cucumis melo, fruit technology, post-harvest management.

\section{Introducción}

En Chile la producción de melón (Cucumis melo L.) con fines de exportación en décadas pasadas fue significativa, llegándose a exportar más de 22.000 t hace cuarenta años, principalmente cultivares del Grupo inodorus debido a su alto potencial de conservación (Krarup, 1984). A partir de esa época las exportaciones tuvieron una clara declinación, alcanzándose valores de sólo 596 t como promedio anual durante los últimos cinco años (ODEPA, 2007). Razones técnicas y comerciales explican el descenso de las exportaciones de melones chilenas.

Recibido 16 noviembre 2007. Aceptado 21 enero 2008

${ }^{1}$ Dirigir correspondencia a C. Krarup: ckrarup@uc.cl
Técnicamente, la exportación disminuyó por la escasa investigación local en factores de producción y de conservación, incluyendo una casi nula evaluación de nuevos cultivares, y por la aparición de enfermedades virales, especialmente del mosaico de la sandía (Watermelon mosaic virus 2, WMV2), enfermedad que limitó notablemente las exportaciones de cultivares tradicionales como Honey Dew y Tendral Amarillo, cuyos frutos se afectan con manchas muy notables que reducen considerablemente la calidad del fruto (Giaconi y Escaff, 2004; Apablaza, 2000). Comercialmente, la exportación de melones tipo inodoro se justificó debido a que estos melones presentan el mayor potencial de conservación (Kader, 2002; Robinson y Decker-Walters, 1999). Sin embargo, los mercados son más 
restringidos, los volúmenes demandados son menores y el precio unitario por kilo es bajo. A esto se sumó la emergencia de otros países competidores, más cercanos a los mercados del hemisferio norte, y al mismo tiempo la competencia de otras frutas de exportación, provocaron una progresiva disminución de este negocio.

En contraste, los melones reticulados (C. melo Grupo Cantalupensis) son los dominantes y más atractivos para la mayoría de los mercados (Robinson y Decker-Walters, 1999). Por ejemplo en Estados Unidos, el consumo de melones reticulados es 4,5 veces superior al de los melones tipo inodoros (Lucier y Jerardo, 2007). La preferencia por estos melones se debe a sus características organolépticas. La mayoría tiene pulpa atractiva de color salmón, indicador de un alto contenido del antioxidante $\beta$-caroteno. La pulpa no se pardea como producto procesado fresco, generando una demanda creciente por estos frutos. Sin embargo, el potencial de conservación de los melones reticulados es menor que el de los melones inodoros (Kader, 2002). Esto afectó su exportación cuando la cantidad de cultivares era limitada y el transporte marítimo era más lento y poco frecuente.

La ampliación de la oferta en el mercado interno del melón y la recuperación de las exportaciones requieren evaluar cultivares de reciente creación, incluyendo aquellos de interés para nuevos mercados. Para esto es necesario investigar los factores de producción más apropiados, y desarrollar las estrategias comerciales para abordar los mercados potenciales. La capacidad de conservación de los melones reticulados ha sido mejorada. Estudios recientes demuestran la existencia de cultivares que permitirían acceder desde Chile, por vía marítima, al mercado de Estados Unidos con un producto de calidad (Krarup y González, 2005; Tohá y Krarup, 2004). Por otro lado, los acuerdos económicos de Chile con otros países obligan a explorar las posibilidades comerciales del melón en mercados como Corea, Japón o Singapur, donde los melones reticulados tienen gran importancia, ya sea como producto fresco o congelado.
Por ejemplo, los melones reticulados son habitualmente consumidos en Japón. Además, son un símbolo de aprecio y se usan como regalo, alcanzando elevados precios que reflejan los complejos métodos utilizados para su producción y comercialización en un nicho muy exclusivo (Sakata y Sugiyama, 2002). Los cultivares utilizados con este propósito corresponden a lo que se denomina melón reticulado tipo Oriental (C. melo Grupo Cantalupensis). Estos cultivares han sido obtenidos y usados en Japón, y otros países asiáticos, tras un prolongado mejoramiento a partir de un germoplasma muy diverso (Fujishita y Masuda, 1994).

Los cultivares más usados en Japón se agrupan en cuatro tipos, siendo dos los tipos más demandados: a. Melones con frutos redondos a ovalados, con epidermis de color verde esmeralda, un reticulado fino y poco denso, de pulpa verde, y b. Melones con frutos casi redondos, con una epidermis de color gris a amarillento, con un reticulado más denso y grueso, de pulpa naranja (Sakata y Sugiyama, 2002). La mayor parte de estos cultivares tienen frutos con alto contenido de sólidos solubles totales (SST), 13 a 14\% como valores mínimos dependiendo de la época de producción (Fujishita y Masuda, 1994). Los melones reticulados cultivados en Chile presentan contenidos de SST más bajos, con valores promedios de 10,5 a 10,9\% (Krarup y González, 2005; Tohá y Krarup, 2004).

Ante el desconocimiento que existe en Chile del melón tipo Oriental y de su potencial como producto fresco, o como materia prima para la industria de procesados frescos y de congelados, esta investigación tuvo como objetivo caracterizar dos cultivares de melones reticulados del tipo Oriental, determinando las principales características cuantitativas y cualitativas de su producción, y el comportamiento durante su almacenamiento refrigerado.

\section{Materiales y métodos}

\section{Precosecha}

La producción de melones cvs. Emerald y Glamour (Sakata Seeds) se realizó en Curacaví 
(33 $\left.26^{\prime} \mathrm{S} ; 7^{\circ} 01^{\prime} \mathrm{O}\right)$ sembrando a inicios de primavera (3 de octubre de 2006) en bandejas modulares de poliestireno. Las plántulas al estado de dos hojas verdaderas (31 de octubre de 2006) fueron trasplantadas en hileras distanciadas a $1,8 \mathrm{~m}$ entre sí, con las plantas separadas a $0,33 \mathrm{~m}$ sobre las hileras (16.666 plantas'ha $\left.{ }^{-1}\right)$. Las labores culturales se realizaron de acuerdo con lo indicado para este cultivo en Chile (Giaconi y Escaff, 2004), difiriendo de lo acostumbrado para estos cultivares en Japón, donde se cultivan en invernaderos, se podan y se ralean los frutos (Fujishita y Masuda, 1994).

\section{Cosecha}

La cosecha se inició a los 132 días desde la siembra, lo que demuestra que estos cultivares son tardíos en comparación a los melones reticulados habitualmente cultivados en Chile (Tohá y Krarup, 2004). El periodo de cosecha se extendió por 18 días. El índice de cosecha para el cv. Emerald fue un cuarto de desprendimiento del pedúnculo y el cv. Glamour se cosechó basado en la apariencia externa de los frutos a los 56 días después de antesis. Sólo se midieron los frutos considerados comerciales con más de $0,6 \mathrm{~kg}$, descartándose los de menor peso, defectuosos, o desprendidos de la planta al momento de la cosecha.

\section{Poscosecha}

Los frutos fueron trasladados de inmediato al laboratorio donde fueron lavados con una solución de hipoclorito de sodio (100 ppm) y secados a temperatura ambiente. Se seleccionaron 30 melones de cada cultivar para las mediciones al momento de cosecha y conservación en poscosecha. El mismo día de cosecha 10 frutos de cada cultivar se caracterizaron respecto de: a. Diámetro ecuatorial y polar $(\mathrm{cm})$, b. Color externo (escala de color), c. Descripción del reticulado (escalas de densidad y grosor), d. Descripción de la pulpa (escala de color, y grosor en $\mathrm{cm}$ ), e. Contenido de sólidos solubles (refractómetro Atago, \%) y firmeza de la pulpa (penetrómetro Effe-Gi, con punta hemisférica de $14 \mathrm{~mm}$, valores convertidos a Newton).

Los melones restantes se separaron en dos grupos de 10 unidades por cultivar y se almacenaron separadamente a 0 y $10^{\circ} \mathrm{C}$, dentro de bolsas plásticas selladas $(0,3 \times 0,4 \mathrm{~m})$, con cuatro perforaciones de $0,5 \mathrm{~cm}$ de diámetro, durante 18 días. Posteriormente se mantuvieron 3 días adicionales a $20^{\circ} \mathrm{C}$ para simular un periodo de comercialización, configurando un tiempo total de almacenamiento de 21 días.

Después de 18 días de conservación a 0 o $10^{\circ} \mathrm{C}$ y antes de ubicarlos en la cámara de conservación a $20^{\circ} \mathrm{C}$, los frutos fueron retirados de la bolsa plástica, estabilizados a temperatura ambiente para eliminar efectos de humedad superficial y se registró el peso de cada fruto. Luego se realizó evaluación visual de daño por enfriamiento (DPE), enfermedades y calidad visual mediante las siguientes escalas (Kader et al., 1973): a. DPE en escala de $1=$ ninguno $(0 \%), 2=$ leve $(\leq 10 \%), 3=$ moderado $(11-30 \%)$, $4=$ severo $(31-50 \%)$ y $5=$ extremo $(>50 \%)$. b. Enfermedades en escala de $1=$ sano, $3=$ leve, podría complicar la venta del fruto, $5=$ moderada, fruto objetable se complica la venta, 7 = severa, normalmente fruto no comerciable y $9=$ extrema, fruto inútil. c. Calidad en escala visual de 1 = inútil, fruto de desecho, $3=$ escasa, límite de venta, $5=$ regular, defectos leves a moderadamente objetables del fruto, 7 = buena, defectos menores del fruto y $9=$ excelente, frutos sin defectos.

$\mathrm{Al}$ término de los tres días adicionales a $20^{\circ} \mathrm{C}$, los frutos fueron reevaluados de la manera ya descrita. Además, se midió el contenido de SST y la firmeza, en forma similar al día de cosecha.

\section{Diseño y análisis estadísticos}

En la ejecución del cultivo se usó un diseño experimental de bloques completamente al azar para los tratamientos (cultivares), con cuatro repeticiones y la unidad experimental consistió en parcelas de $5,94 \mathrm{~m}^{2}$ con diez plantas cada una. En los estudios de poscosecha los tratamientos cultivares, temperaturas y periodos de conservación se distribuyeron en forma complemente al azar con diez repeticiones, siendo la unidad experimental un fruto de melón. Se realizó análisis de varianza (ANDEVA) y los promedios se separaron de acuerdo con la 
prueba de comparación múltiple de Tukey ( $\mathrm{p} \leq$ $0,05)$.

\section{Resultados y discusión}

\section{Características de los frutos en cosecha}

Cosecha. Según lo recomendado para lograr una máxima conservación en melones reticulados, el cv. Emerald se cosechó con un cuarto de desprendimiento del pedúnculo, con un periodo promedio de 51 días desde la antesis, periodo que está dentro del rango de 45 a 55 días citado para estos melones en Japón (Fujishita y Masuda, 1994). La determinación del momento óptimo de cosecha del cv. Glamour fue complejo. Estos frutos, a pesar de tener un reticulado bien formado a los 57 a 65 días desde la antesis, no llegaron a separarse naturalmente de la planta como ocurre con otros melones reticulados, siendo necesario cortarlos. Esta característica es propia de algunos cultivares de melones orientales y señala la importancia de un conocimiento acabado del cultivar y del desarrollo de sus frutos para determinar el momento óptimo de cosecha (Fujishita y Masuda, 1994).

Rendimiento. Con una población de 16.667 plantas ha $^{-1}$ se obtuvo en promedio de 3,13 frutos. planta $^{-1}$, con un peso promedio de $1.010 \mathrm{~g} \cdot$ fruto $^{-1}$ para el cv. Emerald, y de 2,25 frutos'planta ${ }^{-1}$, con un peso promedio de 1.120 $\mathrm{g} \cdot$ fruto $^{-1}$ para el cv. Glamour. Esto significó rendimientos de 42,6 y 53,4 tha ${ }^{-1}$ para Glamour y Emerald, respectivamente. Estos rendimientos fueron mayores que los de otros melones reticulados habitualmente cultivados en Chile. Por ejemplo, melones Colima y Hy Mark, rinden 31,6 y 22,5 tha ${ }^{-1}$ respectivamente (Tohá y Krarup, 2004). Estos rendimientos podrían variar según sea el objetivo de la producción (fresco, procesado fresco o congelado) y con las técnicas de cultivo (poda, raleo, tutorado y riego) usadas.

Calidad visual. Ambos cultivares al momento de cosecha mostraron frutos atractivos,

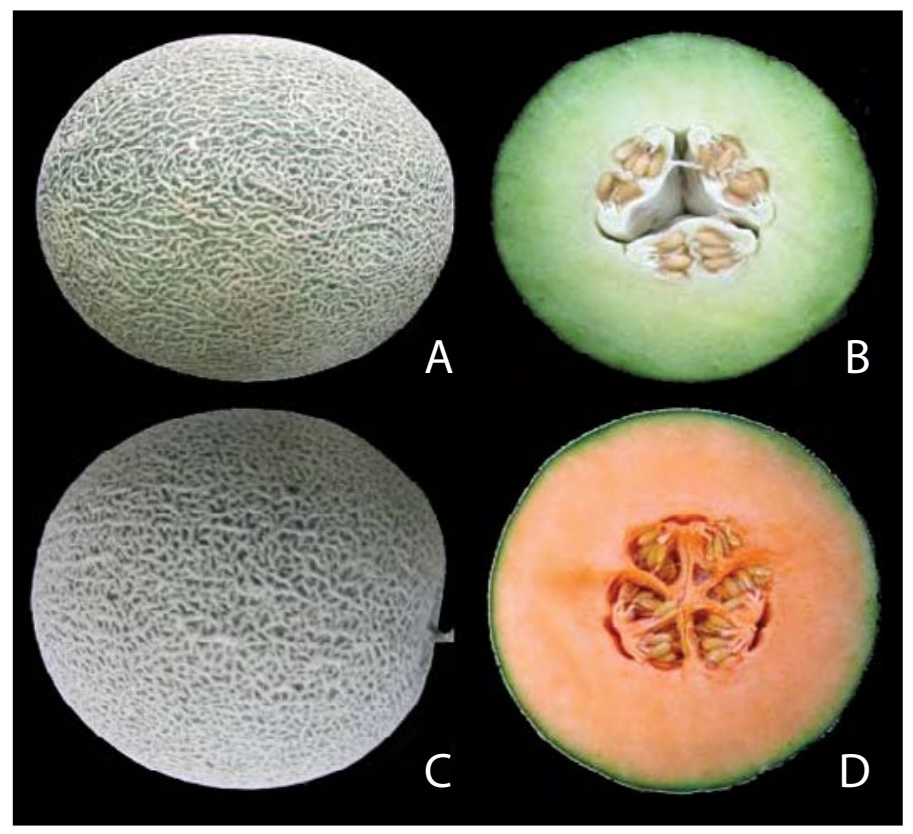

Figura 1. Características externas e internas de los frutos de melón (Cucumis melo) cv. Emerald (A,B) y cv. Glamour (C,D) al momento de cosecha.

Figure 1. External and internal characteristics of melon fruits (Cucumis melo) cv. Emerald (A,B) and cv. Glamour (C,D) at harvest time. 
Cuadro 1. Características externas e internas de melones (Cucumis melo Grupo Cantalupensis) cvs. Emerald and Glamour determinadas a la cosecha.

Table 1. External and internal characteristics of melon fruits (Cucumis melo Grupo Cantalupensis) cvs. Emerald and Glamour evaluated at harvest.

\begin{tabular}{lcc}
\hline & & Cultivares \\
\cline { 2 - 3 } Características & Emerald & Glamour \\
\hline Forma del fruto & Ovalada & Esférico \\
Densidad del reticulado & Bajo a moderado & Alto \\
Grosor del reticulado & Fino & Grueso \\
Intensidad mancha basal & Moderado a alto & Bajo a moderado \\
Color epidermal & Verde esmeralda & Pardo verdoso \\
Color de la pulpa & Verde blanquecino & Salmón intenso \\
Grosor de la pulpa, cm & $3,5 \pm 0,3$ & $4,1 \pm 0,3$ \\
Diámetro ecuatorial, cm & $12,8 \pm 0,9$ & $13,0 \pm 0,5$ \\
Diámetro polar, cm & $13,8 \pm 0,8$ & $13,5 \pm 0,9$ \\
Diámetro cavidad seminal, cm & $5,8 \pm 0,7$ & $4,8 \pm 0,7$ \\
Calidad visual1 & 9,0 & 9,0 \\
Solidos solubles totales, $\%$ & $9,7 \pm 1,3$ & $12,4 \pm 0,6$ \\
Firmeza, $N$ & $28,0 \pm 8,7$ & $18,7 \pm 6,4$ \\
\hline
\end{tabular}

'Escala visual de calidad donde 1 = inútil, 3 = escaso, limita la comercialización; 5 = regular, frutos con defectos leves a moderados, objetables; 7 = bueno, frutos con defectos menores y $9=$ excelente, frutos sin defectos (Kader et al., 1973).

${ }^{\prime}$ Visual quality scale of 1 useless, waste fruit, $3=$ scarce, limited fruit sale, $5=$ regular, light defects to moderately objectionable fruits, $7=$ good, minor fruit defects and $9=$ excellent, fruits without defects (Kader et al., 1973).

uniformes, con una calidad visual excelente, y con sólo escasas imperfecciones (Figura 1). Según la descripción de Sakata y Sugiyama (2002), el cv. Emerald correspondería al Grupo 1 (Earl's Type, melones reticulados de pulpa verde), y el cv. Glamour estaría en el Grupo 2 (Hokkaido King Type, melones reticulados de pulpa naranja), siendo éstos los dos grupos de mayor popularidad en Japón.

Características externas e internas. El cv. Emerald presentó frutos ovalados, con un fondo epidermal verde esmeralda, un reticulado fino y poco denso e internamente, la pulpa fue verde claro, con una cavidad seminal pequeña (Cuadro 1). El cv. Glamour presentó frutos casi esféricos, con un fondo epidermal pardo o verdoso, un reticulado grueso y denso e internamente, la pulpa fue naranja a salmón, con una cavidad seminal muy pequeña (Cuadro 1). El cv. Glamour tiene características parecidas a los melones reticulados de cultivo habitual en Chile. El contenido de SST fue similar en el cv. Emerald al de otros melones reticulados cultivados en Chile (Tohá y Krarup, 2004).

El cv. Glamour presentó un contenido de SST superior a lo habitual. Sin embargo, los valores obtenidos fueron inferiores a los citados en la literatura para este tipo de cultivares (Fujishita y Masuda, 1994). Esto se podría deber a factores ambientales $\mathrm{y}$, especialmente, al manejo del cultivo, ya que no se reguló la carga de frutos por planta. En cuanto a la firmeza del fruto, el cv. Emerald presentó valores superiores al cv. Glamour y, en general, estos melones fueron similares a otros cultivares de melón reticulado producidos en Chile (Tohá y Krarup, 2004).

\section{Características de los frutos en poscosecha}

Pérdida de peso fresco. En ambos cultivares la pérdida de peso fresco fue baja en el periodo de 18 días a $0^{\circ} \mathrm{C}(<1 \%)$ o $10^{\circ} \mathrm{C}(<2 \%)$, con diferencias significativas entre temperaturas de conservación, siendo mayor la pérdida de peso a $10^{\circ} \mathrm{C}$ que a $0^{\circ} \mathrm{C}$ (Figura 2). La exposición de los frutos sin envase a $20^{\circ} \mathrm{C}$ incrementó la tasa de pérdida de peso fresco, alcanzando valores máximos de $5 \%( \pm 0,5 \%)$ para el cv. Emerald y de $4 \%( \pm 0,5 \%)$ para el cv. Glamour. Este último cultivar siempre presentó pérdidas de peso fresco levemente inferior al cv. Emerald. Estudios en melones reticulados tipo Westen Shipper (Tohá y Krarup, 2004) y tipo Galia (Krarup y González, 2005) habitualmente 
muestran valores levemente superiores en situaciones comparables. Los valores de disminución de peso obtenidos no generaron síntomas evidentes en los frutos.

Daño por enfriamiento. En ambos cultivares, la sensibilidad a este desorden fue baja con un daño visible inferior a $10 \%$ de la superficie de los frutos. No obstante, el nivel de daño del cv. Emerald fue mayor al daño del cv. Glamour (Cuadro 2). En el caso del cv. Emerald se observó mayor daño en los frutos almacenados a $10^{\circ} \mathrm{C}$ que a $0^{\circ} \mathrm{C}$, al final del experimento. Esto posiblemente se debió a la mayor pérdida de peso, mayor incidencia de enfermedades y la mayor velocidad en la expresión de síntomas que ocasionalmente ocurren a temperaturas más altas (Kader, 2002). En el caso del cv. Glamour no se observó una diferencia significativa entre tratamientos e independientemente de la temperatura de conservación, los frutos expresaron muy bajo daño.
Daño por enfermedades. La presencia de pudriciones fungosas fue leve y mayor en los frutos almacenados a $10^{\circ} \mathrm{C}$. Entre cultivares, Emerald presentó mayor incidencia de enfermedades a $10^{\circ} \mathrm{C}$, mientras que a $0^{\circ} \mathrm{C}$ no se observaron diferencias significativas entre cultivares (Cuadro 2). Aunque la severidad de las enfermedades fue leve, su presencia constituiría una limitación para la venta de los frutos y debieran aminorarse con tratamientos adecuados.

Sólidos solubles totales. Los valores promedio de SST determinados fluctuaron entre 9,7 y $11,0 \%$ para el cv. Emerald y entre 10,2 y 12,4\% para el cv. Glamour(Cuadros 1 y 2). Si bien estos valores resultan medios a altos al compararlos con los valores habituales que se obtienen con otros melones reticulados en Chile (Krarup y González, 2005; Tohá y Krarup, 2004), están lejos de los 13 a $14 \%$ citados como mínimos para este tipo de melones en Japón (Fujishita

Cuadro 2. Características de poscosecha de melones(Cucumismelo Grupo Cantalupensis) cvs. Emerald and Glamourdespués de 18 días de conservación a 0 o $10^{\circ} \mathrm{C}$ más 3 días adicionales a $20^{\circ} \mathrm{C}$ para simular un periodo de comercialización.

Table 2. Post-harvest characteristics of melons (Cucumis melo Grupo Cantalupensis) cvs. Emerald and Glamour, after 18 days of storage at $0^{\circ}$ or $10^{\circ} \mathrm{C}$ followed by 3 days at $20^{\circ} \mathrm{C}$ to simulate a commercialization period.

\begin{tabular}{|c|c|c|c|c|}
\hline \multirow[b]{2}{*}{ Características } & \multicolumn{4}{|c|}{ Condiciones de almacenaje } \\
\hline & $18 \mathrm{~d} \mathrm{a} 0^{\circ} \mathrm{C}$ & $\begin{array}{r}18 \mathrm{da} 0^{\circ} \mathrm{C} \\
+3 \mathrm{da} 20^{\circ} \mathrm{C}\end{array}$ & 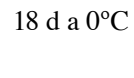 & $\begin{array}{r}18 \mathrm{~d} \mathrm{a} 0^{\circ} \mathrm{C} \\
+3 \mathrm{~d} \mathrm{a} 20^{\circ} \mathrm{C}\end{array}$ \\
\hline \multicolumn{5}{|l|}{ Melon cv. Emerald: } \\
\hline Firmeza, N & nd & $30,7 \pm 6,2$ & nd & $5,8 \pm 1,9$ \\
\hline Sólidos solubles totales, \% & nd & $11,0 \pm 1,3$ & nd & $10,2 \pm 1,3$ \\
\hline Incidente de pudriciones ${ }^{1}$ & $1,2 \pm 0,6$ & $2,4 \pm 0,7$ & $3,8 \pm 1,0$ & $4,3 \pm 2,2$ \\
\hline Daño por frío ${ }^{2}$ & $1,2 \pm 0,2$ & $1,7 \pm 0,5$ & $1,2 \pm 0,3$ & $2,6 \pm 0,9$ \\
\hline Calidad visual $^{3}$ & $8,4 \pm 0,9$ & $6,0 \pm 1,7$ & $6,8 \pm 0,6$ & $4,4 \pm 0,9$ \\
\hline \multicolumn{5}{|l|}{ Melones cv. Glamour: } \\
\hline Firmeza, N & nd & $41,8 \pm 24,4$ & nd & $16,0 \pm 8,5$ \\
\hline Sólidos solubles totales, \% & nd & $11,4 \pm 1,8$ & nd & $10,5 \pm 1,2$ \\
\hline Incidente de pudriciones ${ }^{1}$ & $1,0 \pm 0,0$ & $1,8 \pm 1,4$ & $2,0 \pm 0,6$ & $4,4 \pm 0,9$ \\
\hline Daño por frío ${ }^{2}$ & $1,0 \pm 0,0$ & $1,4 \pm 0,4$ & $1,2 \pm 0,4$ & $1,3 \pm 0,3$ \\
\hline Calidad visual $^{3}$ & $9,0 \pm 0,0$ & $8,4 \pm 0,9$ & $7,0 \pm 0,9$ & $6,2 \pm 1,4$ \\
\hline
\end{tabular}

${ }^{1}$ Escala de enfermedad donde 1 = sano, 3 = leve, dificulta la comercialización, 5 = moderado, fruto objetable difícil de vender; 7 = severo, fruto no comercializable y $9=$ extrema, frutos desechables (Kader et al., 1973).

${ }^{2}$ Escala de daño por frío donde $1=$ ninguno $(0 \%), 2=$ leve $(£ 10 \%), 3=$ moderado $(11-30 \%), 4=\operatorname{severo~}(31-50 \%)$ y $5=$ extremo $(>50 \%)$ (Kader et al., 1973)

${ }^{3}$ Escala visual de calidad donde 1 = inservible, fruto de deshecho, 3 = escaso, limita la comercialización, 5 = regular, defectos leves a moderados, frutos objetables; $7=$ bueno, frutos con defectos menores y $9=$ excelente, frutos sin defectos $($ Kader et al ., 1973). nd $=$ no determinado.

${ }^{1}$ Disease scale of $1=$ healthy, $3=$ light, it may complicate to sale the fruits, $5=$ moderate, objectionable fruits difficult to sell, $7=$ severe, non-commercial fruits, and $9=$ extreme, useless fruits (Kader et al., 1973).

${ }^{2}$ Cooling damage scale of $1=$ none $(0 \%), 2=$ light $(£ 10 \%), 3=$ moderate $(11-30 \%), 4=$ severe $(31-50 \%)$, and $5=$ extreme $(>50 \%)($ Kader et al., 1973).

${ }^{3}$ Visual quality scale of 1 useless, waste fruits, $3=$ scarce, limited fruit sale, $5=$ regular, light defects to moderately objectionable fruits, 7 $=$ good, minor fruit defects and $9=$ excellent, fruits without defects (Kader et al., 1973). nd = not determined . 



Figura 2. Pérdida de peso fresco de frutos de melón (Cucumis melo) cv. Emerald (A) y cv. Glamour (B) después de almacenamiento por 18 días a 0 o $10^{\circ} \mathrm{C}$ y tres días adicionales a $20^{\circ} \mathrm{C}$ para simular un periodo de comercialización.

Figure 2. Fresh weight loss of melon (Cucumis melo) fruits cv. Emerald (A) and cv. Glamour (B) after 18 days storage at o or $10^{\circ} \mathrm{C}$, followed by three days at $20^{\circ} \mathrm{C}$ to simulate a commercialization period.

y Masuda, 1994). Es posible que prácticas de manejo como tutorado, poda, raleo de frutos o restricción del riego, habituales en Japón expliquen estas diferencias (Fujishita y Masuda, 1994).

Firmeza. Independientemente del cultivar, la firmeza se mantuvo más alta en los frutos almacenados a $0^{\circ} \mathrm{C}$ que a $10^{\circ} \mathrm{C}$, lo que estuvo de acuerdo con la más lenta maduración esperada a baja temperatura (Kader, 2002). Al final del período de almacenamiento a $0^{\circ} \mathrm{C}$ no se observaron diferencias entre cultivares. A $10^{\circ} \mathrm{C}$ la firmeza fue mayor en melones cv. Emerald que en melones cv. Glamour.

Calidad visual. Inicialmente la calidad visual de los frutos fue excelente, disminuyendo progresivamente en ambos cultivares durante el almacenamiento. Después de 18 días de conservación, los frutos de los dos cultivares presentaron una mejor calidad visual a 0 que a $10^{\circ} \mathrm{C}$ (Cuadro 2). Estos resultados se deberían a un menor desarrollo de pudriciones, menor desarrollo de síntomas de DPE y menor pérdida de peso fresco a $0^{\circ} \mathrm{C}$ que a $10^{\circ} \mathrm{C}$. $\mathrm{Al}$ comparar los cultivares, los melones cv. Glamour presentaron la mejor calidad visual al final del período de almacenamiento. Luego de 21 días, los melones cv. Emerald almacenados a $10^{\circ} \mathrm{C}$ se encontraron en el límite de venta. En contraste, los melones cv. Glamour, excepto por la presencia de defectos menores, tuvieron una buena calificación, a pesar que algunos frutos se encontraron internamente sobre maduros, posiblemente debido al desconocimiento inicial del momento óptimo de cosecha. Previamente, se ha indicado una duración entre 10 y 21 días para los melones reticulados. Por lo tanto, se puede señalar que los melones cv. Glamour presentan un alto potencial de conservación (Kader, 2002, Shellie y Lester, 2006). 


\section{Resumen}

Este estudio tuvo por objetivo caracterizar el comportamiento en pre y poscosecha de dos cultivares de melón reticulado tipo Oriental (Cucumis melo Grupo Cantalupensis), Emerald y Glamour, producidos bajo prácticas de cultivo habituales en Chile. Ambos cultivares presentaron un desarrollo vigoroso, sin problemas sanitarios y una alta producción de frutos. Los frutos del cv. Emerald desarrollaron una zona de abscisión peduncular y se separaron de la planta al madurar, presentaron tamaño medio $(1,0 \mathrm{~kg})$, forma ovalada, epidermis verde con reticulado fino y poco denso, y pulpa verde cremosa de con 10,3\% como promedio de sólidos solubles totales (SST). En contraste, los frutos del cv. Glamour no desarrollaron una zona de abscisión peduncular y no se separaron de la planta, presentaron tamaño medio $(1,1 \mathrm{~kg})$, con epidermis gris amarillenta con reticulado grueso y denso, y pulpa naranja con mayor contenido de SST $(11,4 \%)$. El rendimiento, tanto en número de frutos cosechados por planta como por unidad de superficie, fue mayor a lo habitual para otros cultivares reticulados en Chile, alcanzando 52,4 th ha ${ }^{-1}$ en el cv. Emerald y 42,4 tha $^{-1}$ en el cv. Glamour. El comportamiento en poscosecha de ambos cultivares fue evaluado después de almacenamiento a 0 y $10^{\circ} \mathrm{C}$ por 18 días, y después de 3 días adicionales a $20^{\circ} \mathrm{C}$. Los resultados indicaron una baja sensibilidad a daños por enfriamiento (DPE) en ambos cultivares, incluso los frutos se conservaron mejor a $0^{\circ} \mathrm{C}$ que a $10^{\circ} \mathrm{C}$, principalmente por la mayor pérdida de peso fresco y la menor incidencia de enfermedades observada a 0 que a $10^{\circ} \mathrm{C}$, lo que resultó en una mejor calidad visual final debido al escaso nivel de DPE observado. El cv. Glamour presentó características más atractivas, una mejor conservación y una mayor calidad final que el cv. Emerald, considerándose un cultivar de gran potencial para consumo fresco y uso industrial.

Palabras claves: Cucumis melo, manejo de poscosecha, tecnología de la fruta.

\section{Literatura citada}

Apablaza, G. 2000. Patología de cultivos. Epidemiología y Control Holístico. Ediciones Universidad Católica de Chile. Santiago, Chile. 347 p.

Fujishita, N. y M. Masuda. 1994. Melons. Pages 7378. In: K. Konishi (ed.). Horticulture in Japan. Asakura Publishing Co. Ltd., Tokio, Japan.

Giaconi, V. y M. Escaff. 2004. Cultivo de Hortalizas. $15^{\mathrm{a}}$ Ed.. Editorial Universitaria. Santiago, Chile, $337 \mathrm{p}$.

Kader, A. 2002. Postharvest Technology of Horticultural Crops. (ed.). Third Ed.. University of California. Agriculture and Natural Resources Publication 3311. CA, USA. 535 p.

Kader; A., L.L. Morris y W.J. Lipton. 1973. Systems for scoring quality of harvested lettuce. HortScience 8:408-409.

Krarup, C. 1984. Análisis de las exportaciones de hortalizas al estado fresco. El Campesino (Chile) 115:20-31.

Krarup, C. y R. González. 2005. El novedoso y promisorio melón Galia. Agronomía y Forestal UC (Chile) 26:20-24.

Lucier, G. y A. Jerardo. 2007. Vegetables and Melons Outlook. United States Department of Agriculture, Economic Research Service, VGS $320.49 \mathrm{p}$.

ODEPA. 2007. Base de Datos. Comercio Exterior. Oficina de Estudios y Planificación Agrícola. Ministerio de Agricultura, Gobierno de Chile. Santiago, Chile.www.odepa.gob.cl(Consultado: octubre de 2007).

Robinson, R. y D.S. Decker-Walters. 1999. Cucurbits. CAB Internacional. NY, USA. 226 p.

Sakata, Y. y M. Sugiyama. 2002. Characteristics of Japanese cucurbits. Acta Horticulturae 588:195203.

Shellie, K.C. y G. Lester. 2006. Netted melons. In: K.C. Gross, C.Y. Wang, and M. Salveit (eds.). The Commercial Storage of Fruits, Vegetables and Florist and Nursery Stocks. USDA Agricultural Handbook 66.

Tohá, J. y C. Krarup. 2004. Evaluación en pre y poscosecha de cultivares de melón reticulado. Un ejemplo de generación de buenas prácticas tecnológicas. Agronomía y Forestal UC (Chile) 22:21-25. 